\title{
Knockdown of the novel proteasome subunit Adrm1 located on the 20q13 amplicon inhibits colorectal cancer cell migration, survival and tumorigenicity
}

\author{
WEI CHEN ${ }^{1}$, XIAO-TONG HU ${ }^{2,3}$, QING-LAN SHI ${ }^{2,3}$, FU-BIAO ZHANG ${ }^{2,3}$ and CHAO HE ${ }^{1-3}$ \\ ${ }^{1}$ Department of Colorectal Surgery, ${ }^{2}$ Biomedical Research Center, Sir Run Run Shaw Hospital, Zhejiang University; \\ ${ }^{3}$ Key Laboratory of Biotherapy of Zhejiang Province, Hangzhou, Zhejiang 310016, P.R. China
}

Received October 13,2008; Accepted November 28, 2008

DOI: $10.3892 /$ or_00000254

\begin{abstract}
The novel proteasome subunit Adrml located on the $20 \mathrm{q} 13$ amplicon was differentially expressed in colorectal cancer by semiquantitative RT-PCR. Adrm 1 mRNA was overexpressed in $46.2 \%$ (18/39) colorectal cancer tissues compared to their matched normal mucosa and significantly correlated with lymph node metastasis of colorectal cancer ( $\mathrm{P}=0.037)$. Knockdown of Adrml by shRNA in human colon carcinoma RKO cells inhibited their anchorage-independent growth, cell migration as well as cell proliferation through inducing apoptosis and cell cycle arrest at the $G_{1}$ phase. In addition, stable RNA interference of Adrml gene synergistic with 5-Fu treatment suppressed RKO cell growth in vitro. Collectively, these data suggested that Adrml is potentially oncogenic and may play an important role in colon tumorigenesis. Regiment with combined application of Adrml RNA interference and chemotherapy may emerge as a novel therapeutic strategy for Adrml overexpressed colorectal cancer.
\end{abstract}

\section{Introduction}

Colorectal cancer (CRC) is one of the most common causes of cancer-related deaths throughout the world. Although advances in treatment have reduced patient morbidity, the survival rates of CRC have remained low. Molecular investigations have provided evidence that multiple genetic alterations are involved in colorectal tumorigenesis. A molecular genetic model of preferential sequence has been proposed (1). However, even with the present knowledge of the cellular and molecular mechanisms of CRC, no biological parameter can predict the behavior of cancers. Karyotypic/cytogenetic data

Correspondence to: Dr Chao He, Department of Colorectal Surgery, Sir Run Run Shaw Hospital, Zhejiang University, Hangzhou, Zhejiang 310016, P.R. China

E-mail: drhe_srrsh@sina.com

Key words: adhesion-regulating molecule 1, shRNA, colorectal cancer, 20q13 amplicon regarding CRC have been accumulated over the last 10 years $(2,3)$, and a gain of $20 q$, especially the $20 q 13$ region, was frequently reported as a common genetic aberration (4-7). Several novel candidate oncogenes have been identified in that region, such as the novel zinc finger gene ZNF217 which is amplified in CRC (8). These data suggest that 20q13 harbors one or more oncogenes which are important for CRC development.

In this study, we found that the novel proteasome subunit Adrm1 located on the 20q13 amplicon was overexpressed in CRCs by semiquantitative RT-PCR. Knockdown of Adrml by shRNA in human colon carcinoma RKO cells inhibited their anchorage-independent growth, cell migration and proliferation. Our results suggested that Adrml is potentially oncogenic and may play an important role in colon tumorigenesis.

\section{Materials and methods}

Patients and tissue samples. A total of 39 frozen tumor and matched normal mucosa blocks (20 Dukes' B, 18 Dukes' C and 1 Dukes' D) retrieved from patients with colorectal adenocarcinoma who underwent surgery from June 2006 to December 2006 at the Sir Run Run Shaw Hospital (Hangzhou, Zhejiang, P.R. China) were investigated in this study. There were 25 male and 14 female patients ranging in age from 40 to 87 years $(61.8 \pm 11.2)$. Patients who received preoperative chemotherapy were excluded from this study.

Cell cultures. All human colorectal cancer cell lines were cultured in Dulbecco's modified Eagle's medium (DMEM) (Gibco BRL, Grand Island, NY) containing 10\% fetal bovine serum (FBS) (Biochrom, Berlin, Germany), $100 \mathrm{U} / \mathrm{ml}$ penicillin (Sigma-Aldrich, St. Louis, MO, USA) and $100 \mu \mathrm{g} / \mathrm{ml}$ streptomycin (Sigma-Aldrich) under humidified conditions in $95 \%$ air and $5 \% \mathrm{CO}_{2}$ at $37^{\circ} \mathrm{C}$.

Semiquantitative RT-PCR. The extraction of total RNA from frozen sections of CRC specimens was carried out using the TRIzol reagent (Invitrogen, Carlsbad, CA, USA) according to the manufacturer's specifications. First-strand complementary DNA (cDNA) synthesis was performed using a GeneAmp RNA PCR core kit (Perkin Elmer, Branchburg, 
NJ). PCR was performed to amplify Adrml with the following primers (Genbank accession no.: NM_007002): forward: 5'-TCA TCC ACC ACC TCT TCC AC-3' and reverse: 5'-CAG ATG GCA AGT AGG GAA GC-3' resulting in a DNA product of $227 \mathrm{bp}$. Each cDNA sample was analyzed in triplicate with the appropriate negative controls.

Construction of Adrml shRNA. The target sequence against human Adrm1 cDNA (NM_007002) was designed at the Ambion website (www.ambion.com) and aligned by BLAST to avoid off-target match. The pSilencer ${ }^{\mathrm{TM}}$ siRNA expression vector kit (Ambion, Austin, TX, USA) was used according to the manufacturer's protocol to produce shRNA expression cassettes (SECs). The most effective SEC targeting Adrml mRNA (5'-GGG CTG GTG TAC ATT CAG C-3') was cloned into the pSilencer ${ }^{\mathrm{TM}}$ 2.1-U6 hygro vector. Cells transfected with the pSilencer hygro plasmid expressing Adrml targetspecific shRNA was compared to cells transfected with the corresponding pSilencer hygro negative control plasmid supplied with the kit.

Cell transfection and stable colony selection. For transfection, RKO cells were plated in 24-well plates at a density of $1 \times 10^{5}$ cells per well and allowed to grow overnight to $90-95 \%$ confluency. The next day, the cells were transfected with the mixture of $0.8 \mu \mathrm{g}$ DNA (Adrml shRNA plasmid or negative control plasmid) and $2 \mu 1$ lipofectamine 2000 (Invitrogen) in $100 \mu 1$ serum-free medium according to the manufacturer's instructions. To produce stably transfected cells, after transfection with the shRNA expression construct, $1500 \mu \mathrm{g} / \mathrm{ml}$ of hygromycin (Roche, Gemany) was added at $48 \mathrm{~h}$ to the medium (DMEM $+15 \%$ FBS). The cells were left in selective medium for two weeks after which they were trypsinized and recultured in selective medium for propagation.

Western blotting. Cell extracts were prepared with lysis buffer. Total protein concentration was measured by using a BCA protein assay reagent kit (Pierce, Rockford, IL, USA). Ten micrograms of sample per well were loaded on a $12 \%$ SDSPAGE and transferred electrophoretically to a nitrocellulose membrane. After blocking in TBS-T buffer with 5\% dry milk for $1 \mathrm{~h}$ at room temperature, the membranes were incubated sequentially with primary antibody overnight at $4^{\circ} \mathrm{C}$ and HRP-conjugated secondary antibody (1:1000, Santa Cruz, CA, USA) for $2 \mathrm{~h}$ at room temperature in TBS-T milk. Primary antibodies were used as follows: anti-Adrm1 mouse monoclonal antibody (3C6, 1:1000, Abnova, Taiwan); antiprocaspase-3 rabbit monoclonal antibody (sc-7148, 1:1000, Santa Cruz Biotechnologies, CA); anti-cleaved caspase- 8 mouse monoclonal antibody (1C12, 1:1000, Cell Signaling, USA) and anti-GAPDH mouse monoclonal antibody (1:1000, Santa Cruz Biotechnologies). Detection was carried out using the ECL kit (Amersham Biosciences, Piscataway, NJ, USA) and the blots were developed using a Fujifilm Las4000 imaging system.

MTT assay. RKO cells were subjected to the MTT [3-(4,5dimethylthiazol-2-yl)-2,5-diphenyltetrazolium bromide] assay, according to the manufacturer's protocol (SigmaAldrich).
TUNEL staining. To detect apoptotic cells, the terminal deoxynucleotidyl transferase-mediated dUTP-biotin nick end-labeling (TUNEL) assay was performed with the In Situ cell death detection kit (Roche, Mannheim, Germany) according to the manufacturer's instructions. Briefly, cells adhered on 6-well plate were fixed in formalin at room temperature, airdried and permeabilized in $0.1 \%$ Triton X-100 and $0.1 \%$ sodium citrate (both Merck, Darmstadt, Germany) for $2 \mathrm{~min}$. After washing in PBS, the sections were incubated with the TUNEL reaction mixture for $90 \mathrm{~min}$ at room temperature. A total number of TUNEL-positive cells derived from five independent high power microscopic fields were summed up, in parallel with that of total cells.

Annexin V-FITC/PI double staining. To measure the numbers and the ratio of apoptotic cells, the Annexin V-FITC/PI apoptosis detection kit (Beyotime, Shanghai, P.R. China) was utilized. The cells were seeded at $1 \times 10^{5} /$ well into 6 -well plates, harvested two days later by trypsinization, washed twice with cold PBS, and then performed according to the manufacturer's instructions.

Cell cycle analysis. Cell cycle analysis was performed with PI. Cells were collected in $5 \mathrm{ml} \mathrm{PBS}$ and spun for $5 \mathrm{~min}$ at $200 \mathrm{x} \mathrm{g}$. Cells were resuspended in $0.5 \mathrm{ml}$ PBS and further fixed in ice-cold $70 \%$ ethanol for at least $2 \mathrm{~h}$. Fixed cells were next centrifuged at $200 \mathrm{x}$ g for $5 \mathrm{~min}$ and the pellet was resuspended in $1 \mathrm{ml}$ of freshly prepared PI staining solution $(20 \mu \mathrm{g} / \mathrm{ml}$ $\mathrm{PI} / 0.1 \%$ Triton/0.2 mg DNase-free RNase A). Samples were kept for $30 \mathrm{~min}$ at room temperature and maintained at $4{ }^{\circ} \mathrm{C}$ overnight in the dark.

Soft agar assay. For the soft agar colony formation assay, $\sim 500$ cells were suspended in medium containing $0.3 \%$ low melt agarose, seeded into a six-well plate that was overlaid with $0.5 \%$ low melt agarose, and allowed to grow for two weeks at $37^{\circ} \mathrm{C}$ in $5 \% \mathrm{CO}_{2}$. The colonies containing $>50$ cells were counted under a microscope. Three to five wells were analyzed for each experiment.

Wound-healing assay. Cell migration was studied by a scratch wound-healing assay. Cells were cultured in a sixwell plate until confluent. The cell layer was wounded using a sterile tip. Photographs of the cells invading the scratch were taken every $24 \mathrm{~h}$. The experiment was done in triplicate.

Cell migration assay. RKO cells transfected with either Adrml shRNA or scrambled shRNA were trypsinized and resuspended in DMEM containing $1 \%$ FBS at a density of $1 \times 10^{6}$ cells $/ \mathrm{ml}$. In all, $100 \mu 1$ of the cell suspension was added into upper chamber of a transwell (Corning, USA) consisted of inserts containing $8-\mu \mathrm{m}$ pore-size PET membrane. DMEM (600 $\mu \mathrm{l})$ containing $10 \%$ FBS was placed in the lower chamber. After incubation for $24 \mathrm{~h}$, cells remained in upper chamber was removed carefully by cotton swab and the membrane was cut off by an operating knife. The side facing lower chamber was stained with $0.05 \%$ crystal violet and attached cells were counted under a light microscope. The experiment was performed 3 times. 
(A)

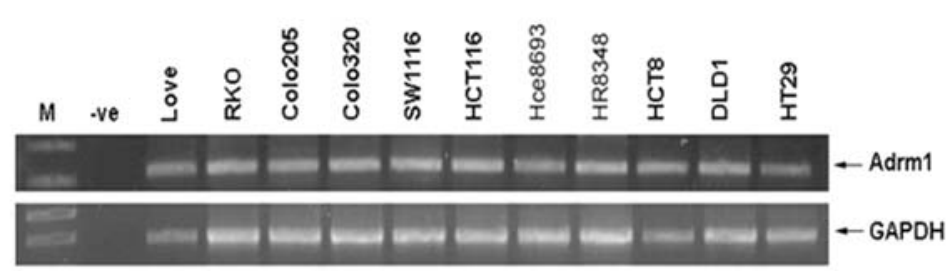

(B)

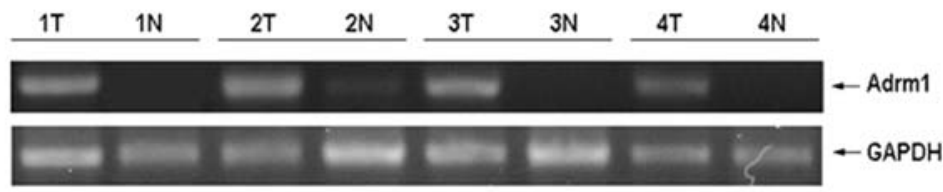

(C)

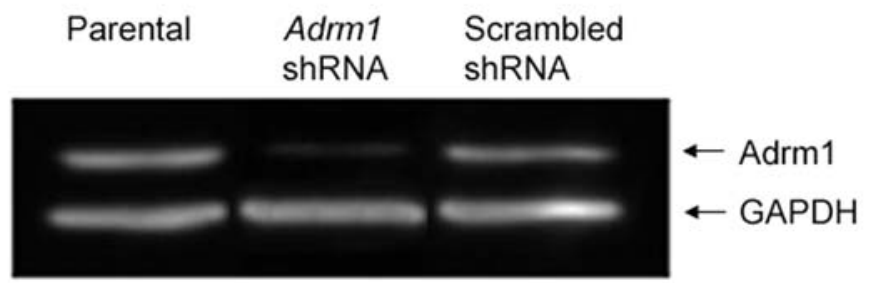

(D)

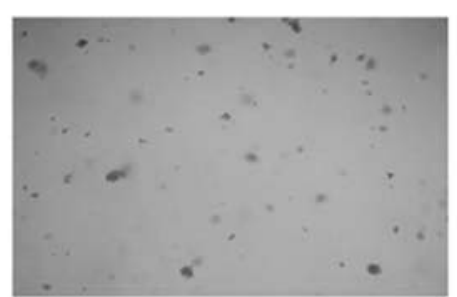

Parental

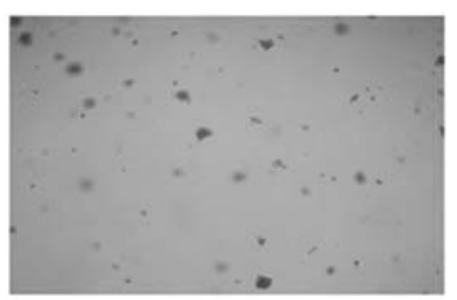

Scrambled shRNA

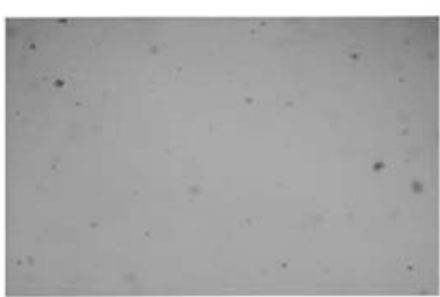

Adrm1 shRNA

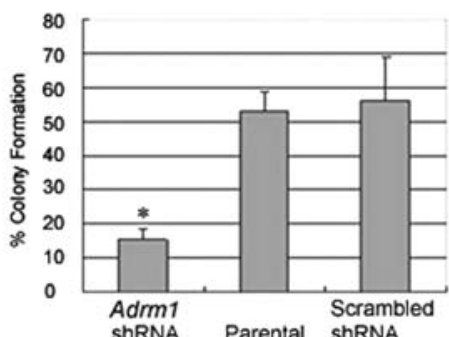

Figure 1. Adrml knockdown suppresses RKO cell growth. (A) Adrml mRNA expression in colorectal cancer cell lines. (B) Representative semiquantitative RT-PCR results of Adrml in colorectal cancer tissues (T) and their matched normal mucosa (N). The expression of GAPDH served as a control; M, marker and -ve, negative control. (C) Western blot analysis showed Adrm1 protein expression level of parental, Adrm1 shRNA transfected and scrambled shRNA transfected RKO cells. (D) The parental, Adrml shRNA transfected and scrambled shRNA transfected RKO cells were assayed for colony formation ( $3 \times 10^{4}$ cells per $60-\mathrm{mm}$ dish) in soft agar. Colonies were counted and photographed after 21 days of growth (original magnification: $\left.\times 400\right)$. These results are representative of three independent experiments. A separate cluster with $>50$ cells was regarded as a clone. Colony formation efficiency (\%) was defined as the percentage of colonies in the cells seeded. Error bars represent the standard error of the mean. ${ }^{~} \mathrm{P}<0.05$ when compared to the control cells.

Statistical analysis. Statistical analysis was performed in SPSS 11.0 for Windows (SPSS Inc., Chicago, IL, USA). The two-tailed Chi-square and Fisher's exact test were used to analyze the association of the Adrml mRNA expression with the different clinicopathological parameters. $\mathrm{P}<0.05$ was considered statistically significant.

\section{Results}

Identification of Adrml mRNA differentially expressed in colorectal cancer versus matched normal mucosa. To search for the Adrml mRNA expression status in colorectal cancer, semiquantitative RT-PCR was performed in 11 colorectal cancer cell lines and 39 CRC cases (RNA quality of these cases was excellent and the amount of cDNA was normalized). Adrml mRNA was expressed in all the cell lines (Fig. 1A) and overexpressed in $46.2 \%$ (18/39) colorectal cancer tissues compared to their matched normal mucosa (Fig. 1B).

The correlations between the clinicopathological parameters of patients with colorectal cancer and the Adrml mRNA overexpression are summarized in Table I. The Adrml mRNA overexpression was significantly correlated with 
Table I. The association between Adrml mRNA expression and clinicopathological parameters in colorectal cancer.

\begin{tabular}{|c|c|c|c|c|}
\hline \multirow[b]{2}{*}{$\begin{array}{l}\text { Clinicopathological } \\
\text { parameters }\end{array}$} & \multirow[b]{2}{*}{$\mathrm{n}=39$} & \multicolumn{2}{|c|}{ Adrml mRNA expression } & \multirow[b]{2}{*}{ P-value } \\
\hline & & $\begin{array}{l}\text { Low } \\
(n=21)\end{array}$ & $\begin{array}{l}\text { High } \\
(\mathrm{n}=18)\end{array}$ & \\
\hline \multicolumn{5}{|l|}{ Sex } \\
\hline Male & 25 & 13 & 12 & 0.757 \\
\hline Female & 14 & 8 & 6 & \\
\hline \multicolumn{5}{|l|}{ Age } \\
\hline$\leq 61.8$ & 17 & 8 & 9 & 0.455 \\
\hline$>61.8$ & 22 & 13 & 9 & \\
\hline \multicolumn{5}{|l|}{ Location } \\
\hline Colon & 17 & 7 & 10 & 0.163 \\
\hline Rectum & 22 & 14 & 8 & \\
\hline \multicolumn{5}{|l|}{ Differentiation } \\
\hline Well + moderate & 28 & 15 & 13 & 0.620 \\
\hline Poor & 11 & 6 & 5 & \\
\hline \multicolumn{5}{|l|}{ Dukes' stage } \\
\hline $\mathrm{A}+\mathrm{B}$ & 20 & 14 & 6 & $0.037^{\mathrm{a}}$ \\
\hline $\mathrm{C}+\mathrm{D}$ & 19 & 7 & 12 & \\
\hline \multicolumn{5}{|l|}{$\begin{array}{l}\text { Lymph node } \\
\text { metastasis }\end{array}$} \\
\hline Positive & 19 & 7 & 12 & $0.037^{\mathrm{a}}$ \\
\hline Negative & 20 & 14 & 6 & \\
\hline
\end{tabular}

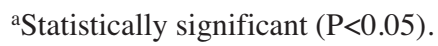

lymph node metastasis $(\mathrm{P}=0.037)$ and Dukes' stage $(\mathrm{A}+\mathrm{B}$ vs. $\mathrm{C}+\mathrm{D}, \mathrm{P}=0.037)$. The Adrml mRNA overexpression had no significant correlation with patient gender, age, tumor location, and differentiation.

Adrm1 knockdown inhibited the proliferation of RKO cells. To reduce Adrml expression, we developed an Adrm 1 shRNA plasmid that caused significant reduction of Adrml expression without any detectable change in $G A P D H$ expression (Fig. 1C). The effect of Adrml knockdown on colony-forming efficiency in soft agar is shown in Fig. 1D. The colony forming ability of parental and scrambled shRNA transfected RKO cells was $\sim 53 \%$ and $56 \%$ respectively while that of Adrml shRNA transfected cells was $\sim 15.5 \%(\mathrm{P}<0.05)$. These results indicated that Adrml gene expression enhanced the growth rate and malignant tendency of cells.

Moreover, the flow cytometry results showed an increase of $\mathrm{G}_{0} / \mathrm{G}_{1}$ phase cells from $37.32 \pm 1.73 \%$ (parental cells) to $41.18 \pm 1.09 \%$ (Adrml shRNA transfected cells), but otherwise an decrease of $\mathrm{S} / \mathrm{G}_{2}$ phase cells from $62.68 \pm 1.70 \%$ to $58.80 \pm 1.10 \%$ (Table II). Adrml knockdown appeared to suppress RKO cell proliferation due to cell cycle arrest at the $\mathrm{G}_{1}$ phase, in other words, Adrml may promote RKO cell proliferation by accelerating cell cycling.
Table II. Adrml knockdown arrested cell cycle at the G1 phase.

\begin{tabular}{lcc}
\hline RKO cells & $\mathrm{G}_{0} / \mathrm{G}_{1}$ & $\mathrm{~S} / \mathrm{G}_{2}$ \\
\hline Parental & $37.32 \pm 1.73$ & $62.68 \pm 1.70$ \\
Scrambled shRNA transfected & $27.66 \pm 0.40$ & $72.32 \pm 0.41$ \\
Adrml shRNA transfected & $41.18 \pm 1.09$ & $58.80 \pm 1.10^{\mathrm{a}}$ \\
\hline
\end{tabular}

${ }^{\mathrm{a}} \mathrm{P}<0.05$ when compared to parental and scramble shRNA transfected cells.

Adrml knockdown increased the spontaneous apoptosis of RKO cells. We utilized TUNEL staining to evaluate DNA damage of the cells. As shown in Fig. 2A, significantly larger number of TUNEL-positive nuclei were observed in Adrml shRNA transfected cells compared with those of parental and scrambled shRNA transfected cells. Quantification of TUNEL-positive nuclei revealed that the fraction of TUNELpositive cells increased by 10 -fold with Adrml shRNA transfected compared with untransfected and scrambled shRNA transfected.

Then, we examined the effects of Adrml knockdown on caspases by Western blotting. Fig. 2B demonstrated that Adrml knockdown induced cleavage (activation) of caspase 8 which produce p43/41 while procaspase-3 was markedly attenuated. Caspase-3 activation plays a key role in initiation of cellular events during the early apoptotic process. It suggested most cells were in the late apoptotic stage. Together, these experiments indicated that Adrml knockdown increased the spontaneous apoptotic responses in RKO cells.

Adrml knockdown suppressed the migration of RKO cells. Comparison of the spindle morphology of parental and scrambled shRNA transfected RKO cells, the Adrml shRNA transfected cells grown at both low and high density were characterized by the deficiency of lamellipodial extensions and displayed rounded morphology resulting in complete detachment from tissue culture dishes (Fig. 3A). This suggested that Adrm1 may play a role in motility-related processes and Adrml knockdown may decrease cell migration speed.

To confirm the correlation between the Adrml knockdown and migration of RKO cells, wound-healing assay was performed. The Adrml shRNA transfected cells took $>48 \mathrm{~h}$ to close a scratch wound, whereas parental and scrambled shRNA transfected cells took $<48 \mathrm{~h}$ to heal a similar size wound (Fig. 3B).

We further examined cell migration of the Adrml shRNA transfected cells by 24-transwell system (Corning). As shown in Fig. 3C, Adrml knockdown led to significant suppression of RKO cell migration after a $24-\mathrm{h}$ period.

Adrml knockdown increased the sensitivity to 5-fluorouracil (5-Fu) in RKO cells. Since 5-Fu is a widely used chemotherapeutic agent during the past two decades in treatment of CRC, we explored the sensitivity of Adrml knockdown RKO cells to 5-Fu treatment. A dose-response curve for 5-Fu 
(A)
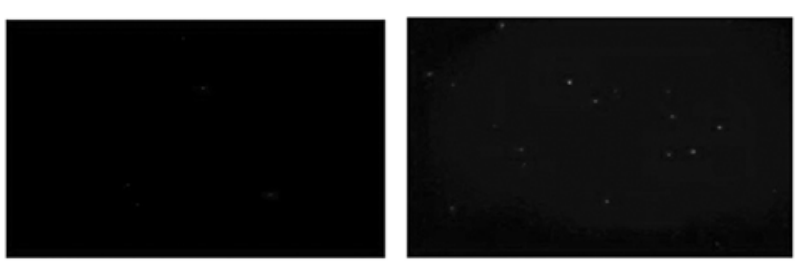

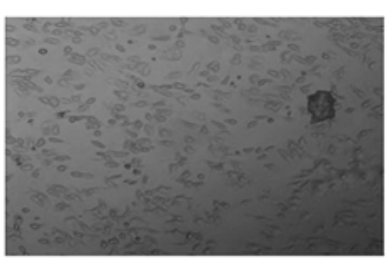

Scrambled shRNA

(B)

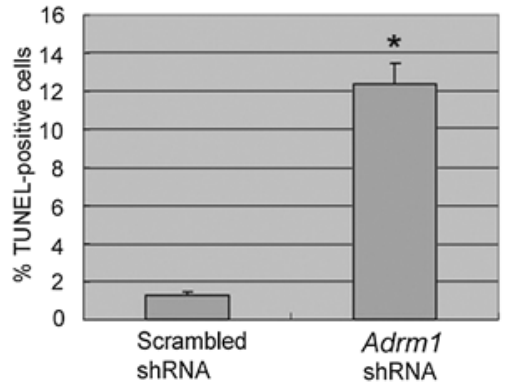

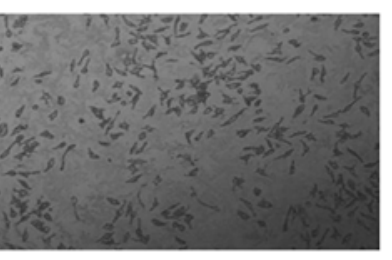

Adrm1 shRNA

(C)

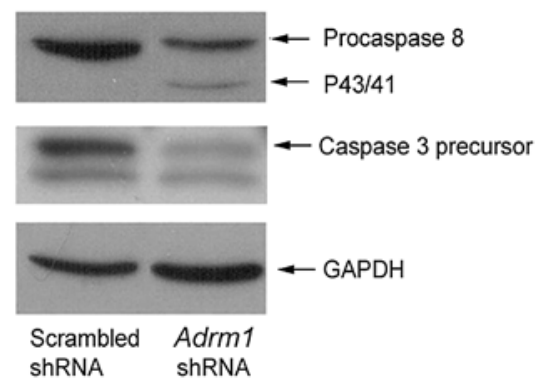

Figure 2. Adrm1 knockdown increased the spontaneous apoptosis of RKO cells (A) Adrml shRNA and scrambled shRNA transfected RKO cells were stained by the TUNEL method to reveal DNA strand breaks indicative of apoptosis. Upper panel: micrographs taken with fluorescent microscopy; Lower panel: micrographs taken with bright field microscopy (original magnification: $\mathrm{x} 400$ ). (B) Quantitation of TUNEL-positive cells $(\mathrm{P}<0.05$ ). The bars indicate $\pm \mathrm{SE}$ from the mean of three separate experiments. (C) Western blotting showed the cleavage of caspase- 8 and -3 in Adrm1 shRNA and scrambled shRNA transfected RKO cells. ${ }^{*} \mathrm{P}<0.05$ when compared to the control cells.

revealed that $\mathrm{IC}_{50}$ on $\mathrm{RKO}$ cells was $12.5 \mu \mathrm{M}$ for $48 \mathrm{~h}$ (data not shown). Then we measured the effect of Adrml knockdown on RKO cell growth using the MTT assay. The Adrm 1 shRNA transfected cells displayed greatly reduced cell numbers (Fig. 3C), indicating apoptotic cell death (Fig. 3D), upon treatment with 5-Fu.

\section{Discussion}

In this study, Adrm1 located on 20q13 amplicon was found to be overexpressed in $46.2 \%$ (18/39) colorectal cancer tissues compared to their matched normal mucosa. This is consistent with previous studies. Simins et al have identified Adrml as a novel cell adhesion-promoting receptor that was upregulated in metastatic cancer cells. Northern blot analysis of human breast cancer cell lines revealed 3- to 5-fold elevated Adrm 1 mRNA levels in metastatic as compared to nonmetastatic cells (9). Moreover, Pilarsky et al identified Adrml as commonly overexpressed gene in solid tumors compared to normal tissues by comparison of microarray data and also validated its differential expression by hybridization of a genespecific probe (10). Recently, Fejzo et al also identified AdrmI as amplification target after comprehensive analysis of 20q13 genes in ovarian cancer. Overexpression of Adrml correlated significantly with shorter time to recurrence and overall survival of ovarian cancer (11).
Adhesion-regulating molecule 1 (Adrm1), also known as GP110, hRpn13 or MGC29536, is located on the $20 \mathrm{q} 13$ amplicon. It is suggested that the chromosome segment $20 \mathrm{q} 13$ may harbor one or more oncogenes that are important for colorectal cancer development. Adrml was described initially as an interferon-inducible membrane glycoprotein with a possible role in cancer (12). Database search analysis suggested that Adrml was a human ortholog of Rpn13, a subunit of budding yeast proteasomes (13). Four independent research groups have demonstrated that Adrml was a novel component of $19 \mathrm{~S}$ regulatory complex of $26 \mathrm{~S}$ proteasome (14-17), directly associated with the deubiquitinating enzyme $\mathrm{UCH} 37$ and anchored this enzyme to the proteasome.

The $26 \mathrm{~S}$ proteasome plays a central role in cell cycle progression, transcription factor activation, apoptosis, and other cellular events (18). Inhibiting proteasome activity leads to cell cycle apoptosis and the deregulation of the proteasome pathway was often associated with cancer-related processes such as oncogenic transformation, tumor progression, escape from immune surveillance and drug resistance (19). More interesting, recently we also found the proteasome subunit PSMA7 located on the 20q13 amplicon was overexpressed and associated with liver metastasis in colorectal cancer (20). The available information suggests that involvement in a malignant phenotype is conceivable for the proteasome subunit Adrml $(9,10)$. 
(A)

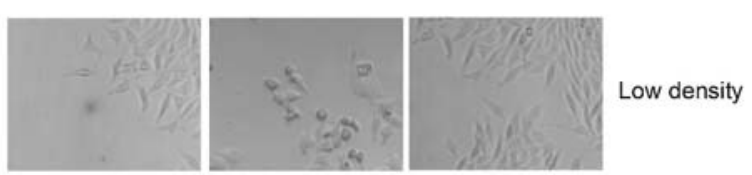

(D)

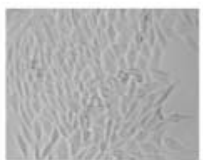

Parental
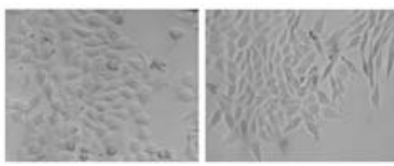

High density

Adrm1 shRNA Scrambled shRNA

(B)
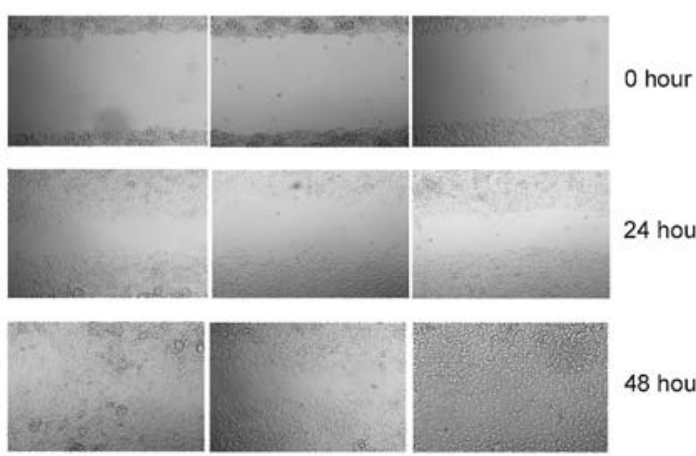

Parental
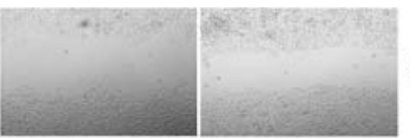

24 hours

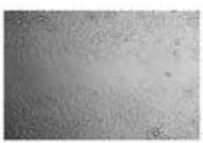

Adrm1 shRNA Scrambled shRNA

(C)
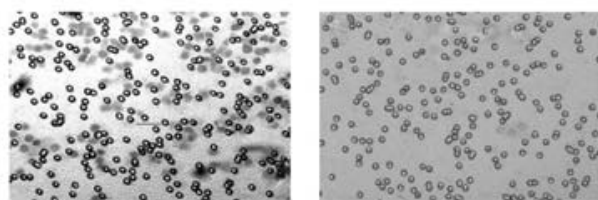

Adrm1 shRNA

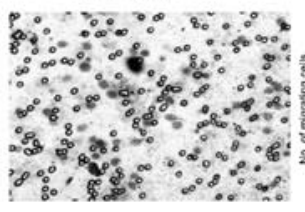

Scrambled shRNA

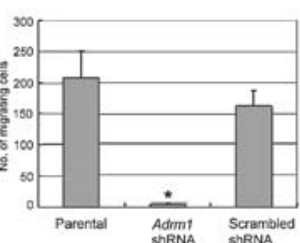

48 hours

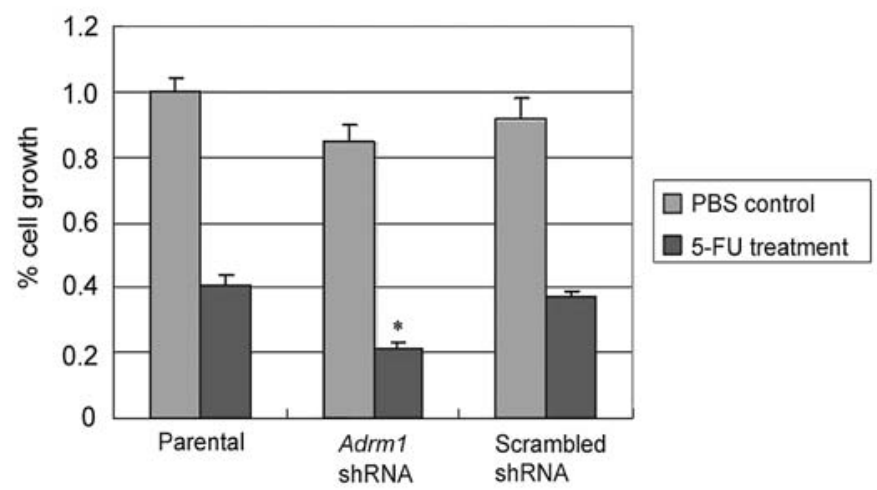

(E)

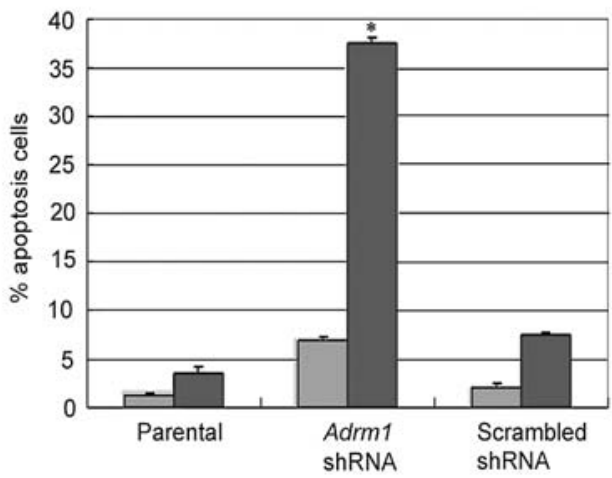

Figure 3. Adrm1 knockdown decreased the migration of RKO cells and increased the sensitivity of RKO cells to 5-Fu treatment. (A) Adrm1 shRNA and scrambled shRNA transfected RKO cells showed substantial morphological changes compared to parental and scrambled shRNA transfected RKO cells (original magnification: x100). Upper panel, low density; lower panel, high density. (B) Wound healing assay for parental, Adrm1 shRNA transfected and scrambled shRNA transfected RKO cells. The images were taken every $24 \mathrm{~h}$ (original magnification: x100). (C) Migration of parental, Adrm 1 shRNA transfected and scrambled shRNA transfected RKO cells were assayed by 24-transwell system. The images of migrated cells were taken $24 \mathrm{~h}$ after seeding (original magnification: x100). The number of cells that had migrated was counted in five representative high power fields (HPFs) per transwell insert. (D) Effect of Adrm1 knockdown on the sensitivity of RKO cell to 5-Fu treatment (MTT assay). (E) Apoptotic cells induced by 48 h 5-Fu (12.5 $\mu$ M) treatment in parental, Adrm1 shRNA transfected and scrambled shRNA transfected RKO cells were detected with Annexin V-FITC/PI double staining. PBS treatment as a control. Error bars represent the standard error of the mean. ${ }^{*} \mathrm{P}<0.05$ when compared to the control cells.

In the present study, we found Adrml overexpression was significantly correlated with lymph node metastasis of colorectal cancer $(\mathrm{P}=0.037)$. Knockdown of Adrml by shRNA inhibited not only anchorage-independent growth but also the migration of RKO cells as well as their proliferation through inducing apoptosis and cell cycle arrest at the $\mathrm{G}_{1}$ phase in vitro. This property may involve interactions of Adrml with Uch37 and proteasomes, although Adrml may have other functions that are yet to be discovered. The molecular mechanisms by which Adrm1 plays a role in colorectal cancer progression will be investigated in detail in a future study.

In summary, we reported the first evidence that the novel proteasome subunit Adrm1 located on the 20q13 amplicon is an important mediator of migration, metastasis in human colorectal cancer cells and also modulates in vitro colorectal cancer cell growth. Additionally, our data showed that Adrml RNA interference combined with 5-Fu treatment efficiently suppressed colorectal cancer cell growth in vitro. Regimen with combined application of Adrml RNAi and chemotherapy may emerge as a novel therapeutic strategy for Adrml overexpressed colorectal cancer.

\section{Acknowledgements}

This work was supported by the Natural Science Foundation of Zhejiang Province, No. Y206090. 


\section{References}

1. Fearon ER and Vogelstein B: A genetic model for colorectal tumorigenesis. Cell 61: 759-767, 1990.

2. Bardi G, Sukhikh T, Pandis N, Fenger C, Kronborg O and Heim S: Karyotypic characterization of colorectal adenocarcinomas. Genes Chromosomes Cancer 12: 97-109, 1995.

3. Bardi G, Johansson B, Pandis N, et al: Cytogenetic aberrations in colorectal adenocarcinomas and their correlation with clinicopathologic features. Cancer 71: 306-314, 1993.

4. Meijer GA, Hermsen MA, Baak JP, et al: Progression from colorectal adenoma to carcinoma is associated with non-random chromosomal gains as detected by comparative genomic hybridization. J Clin Pathol 51: 901-909, 1998

5. Ried T, Knutzen R, Steinbeck R, et al: Comparative genomic hybridization reveals a specific pattern of chromosomal gains and losses during the genesis of colorectal tumors. Genes Chromosomes Cancer 15: 234-245, 1996.

6. Schlegel J, Stumm G, Scherthan H, Bocker T, Zirngibl H, Ruschoff $\mathbf{J}$ and Hofstadter F: Comparative genomic in situ hybridization of colon carcinomas with replication error. Cancer Res 55: 6002-6005, 1995.

7. Lassmann S, Weis R, Makowiec F, Roth J, Danciu M, Hopt U and Werner M: Array CGH identifies distinct DNA copy number profiles of oncogenes and tumor suppressor genes in chromosomal- and microsatellite-unstable sporadic colorectal carcinomas. J Mol Med 85: 293-304, 2007.

8. Rooney PH, Boonsong A, McFadyen MC, McLeod HL, Cassidy J, Curran S and Murray GI: The candidate oncogene ZNF217 is frequently amplified in colon cancer. J Pathol 204: 282-288, 2004

9. Simins AB, Weighardt H, Weidner KM, Weidle UH and Holzmann B: Functional cloning of ARM-1, an adhesionregulating molecule upregulated in metastatic tumor cells. Clin Exp Metastasis 17: 641-648, 1999.

10. Pilarsky C, Wenzig M, Specht T, Saeger HD and Grutzmann R: Identification and validation of commonly overexpressed genes in solid tumors by comparison of microarray data. Neoplasia 6: 744-750, 2004.
11. Fejzo MS, Dering J, Ginther C, et al: Comprehensive analysis of $20 \mathrm{q} 13$ genes in ovarian cancer identifies ADRM1 as amplification target. Genes Chromosomes cancer (In press).

12. Shimada S, Ogawa M, Takahashi M, Schlom J and Greiner JW: Molecular cloning and characterization of the complementary DNA of an M(r) 110,000 antigen expressed by human gastric carcinoma cells and upregulated by gamma-interferon. Cancer Res 54: 3831-3836, 1994.

13. Verma R, Chen S, Feldman R, et al: Proteasomal proteomics: identification of nucleotide-sensitive proteasome-interacting proteins by mass spectrometric analysis of affinity-purified proteasomes. Mol Biol Cell 11: 3425-3439, 2000.

14. Hamazaki J, Iemura S, Natsume T, Yashiroda H, Tanaka K and Murata S: A novel proteasome interacting protein recruits the deubiquitinating enzyme UCH37 to $26 \mathrm{~S}$ proteasomes. EMBO J 25: 4524-4536, 2006.

15. Jorgensen JP, Lauridsen AM, Kristensen P, Dissing K, Johnsen AH, Hendil KB and Hartmann-Petersen R: Adrm1, a putative cell adhesion regulating protein, is a novel proteasomeassociated factor. J Mol Biol 360: 1043-1052, 2006.

16. Qiu XB, Ouyang SY, Li CJ, Miao S, Wang L and Goldberg AL: hRpn13/ADRM1/GP110 is a novel proteasome subunit that binds the deubiquitinating enzyme, UCH37. EMBO J 25: 5742-5753, 2006.

17. Yao T, Song L, Xu W, et al: Proteasome recruitment and activation of the Uch37 deubiquitinating enzyme by Adrm1. Nat Cell Biol 8: 994-1002, 2006.

18. King RW, Deshaies RJ, Peters JM and Kirschner MW: How proteolysis drives the cell cycle. Science 274: 1652-1659, 1996.

19. Spataro V, Norbury C and Harris AL: The ubiquitin-proteasome pathway in cancer. Br J Cancer 77: 448-455, 1998.

20. Hu XT, Chen W, Wang D, et al: The proteasome subunit PSMA7 located on the 20q13 amplicon is overexpressed and associated with liver metastasis in colorectal cancer. Oncol Rep 19: 441-446, 2008. 